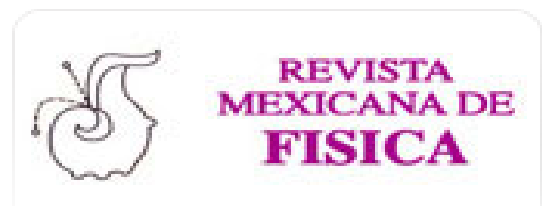

Revista Mexicana de Física

ISSN: 0035-001X

rmf@ciencias.unam.mx

Sociedad Mexicana de Física A.C.

México

Mello, P.A.

Statistical wave scattering: from the atomic nucleus to mesoscopic systems to microwave cavities

Revista Mexicana de Física, vol. 53, núm. 6, -diciembre, 2007, pp. 64-69

Sociedad Mexicana de Física A.C.

Distrito Federal, México

Available in: http://www.redalyc.org/articulo.oa?id=57028300012

How to cite

Complete issue

- More information about this article

Journal's homepage in redalyc.org

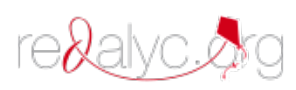

Scientific Information System Network of Scientific Journals from Latin America, the Caribbean, Spain and Portugal Non-profit academic project, developed under the open access initiative 


\title{
Statistical wave scattering: from the atomic nucleus to mesoscopic systems to microwave cavities
}

\author{
P.A. Mello \\ Instituto de Física, Universidad Nacional Autónoma de México, \\ 01000 México Distrito Federal, México.
}

Recibido el 1 de febrero de 2007; aceptado el 24 de agosto de 2007

Universal statistical aspects of wave scattering by a variety of physical systems ranging from atomic nuclei to mesoscopic systems and microwave cavities are described. A statistical model for the scattering matrix is employed to address the problem of quantum chaotic scattering. The model, introduced in the past in the context of nuclear physics, discusses the problem in terms of a prompt and an equilibrated component: it incorporates the average value of the scattering matrix to account for the prompt processes and satisfies the requirements of flux conservation, causality and ergodicity. The main application of the model is the analysis of electronic transport through ballistic mesoscopic cavities whose classical dynamics is chaotic, although it can be applied to the propagation of microwaves through cavities of a similar shape. The model describes well the results from the numerical solutions of the Schrödinger equation for two-dimensional cavities.

Keywords: Statistical wave scattering; compound nucleus; mesoscopic systems; microwave cavities.

Se describen los aspectos universales de la dispersión de ondas por toda una variedad de sistemas físicos, cuya escala que va desde los núcleos atómicos hasta las cavidades de microondas, pasando por los sistemas mesoscópicos. Se emplea un modelo estadístico de la matriz de dispersión para describir el problema de la dispersión caótica cuántica. El modelo, introducido en el pasado en el contexto de la física nuclear, discute el problema en términos de una componente rápida y una equilibrada: incorpora el valor promedio de la matriz de dispersión para describir los procesos rápidos y satisface los requerimientos de conservación de flujo, causalidad y ergodicidad. La principal aplicación del modelo es el análisis del transporte electrónico a través de cavidades mesoscópicas cuya dinámica clásica es caótica, aunque se puede aplicar también de una forma semejante a la propagación de microondas a través de cavidades. El modelo describe bien los resultados de las soluciones numéricas de la ecuación de Schrödinger para cavidades bidimensionales.

Descriptores: Dispersión estadística de ondas; núcleo compuesto; sistemas mesoscópicos; cavidades de microondas.

PACS: 24.60.Dr; 73.23.-b; 73.63.Kv; 41.20.Jb; 84.40.-x

\section{Introduction}

The problem of coherent multiple scattering of waves has long been of great interest in physics and, in particular, in optics. There are a great many wave-scattering problems, appearing in various fields of physics, where the interference pattern due to multiple scattering is so complex, that only a statistical treatment is feasible and, perhaps, meaningful $[1,2]$.

Nuclear physics (with typical dimensions on the scale of the fm) offers very good examples of complex quantummechanical scattering. Indeed, the statistical theory of nuclear reactions has been of great interest for many years, in those cases where, due to the presence of many resonances, the excitation function is very complicated as a function of energy and its detailed structure is of little interest. A statistical treatment is then called for.

Most remarkably, one finds similar statistical properties in the quantum-mechanical scattering of "simple" oneparticle systems -like a particle scattering from a cavitywhose classical dynamics is chaotic. Experimental realizations of these systems are, for instance:

i) microstructures called ballistic quantum dots, whose typical dimensions are of the order of $1 \mu \mathrm{m}$, and

ii) cavities with a similar shape, but now with a typical dimension of $0.5 \mathrm{~m}$, used to scatter microwaves.
The relation of the nuclear problem with the theory of waveguides and cavities was proposed very clearly by Ericson and Mayer-Kuckuk [3] about fourty years ago: "Nuclear-reaction theory is equivalent to the theory of waveguides... We will concentrate on processes in which the incident wave goes through a highly complicated motion in the nucleus... We will picture the nucleus as a closed cavity, with reflecting but highly irregular walls." We now know that the "irregular walls" are not necessary to see these features and that the similarity in the statistical properties of these various physical systems occurs as long as the corresponding classical dynamics is chaotic.

The purpose of this contribution is to review past and recent work in which the ideas behind the nuclear optical model and the statistical theory of the compound nucleus [4] have been used to give a unified treatment of microwave propagation through cavities and of quantum-mechanical scattering in simple one-particle systems in which the corresponding classical dynamics is chaotic (see Ref. [1] and references contained therein).

The most remarkable feature we shall encounter is the statistical regularity of the results: they will be expressible in terms of a few relevant physical parameters, the remaining details being just "scaffoldings". This feature will be captured within a maximum-entropy approach.

In the next section we present some general physical ideas related with scattering of microwaves by classically chaotic 
cavities and of electrons by ballistic quantum dots. The formulation of the scattering problem is given in Sec. 3 where we employ, to be specific, the language of electron scattering. The general ideas behind doing statistics with the scattering matrix and the maximum-entropy model reviewed here are presented in Secs. 4 and 5, respectively. The comparison of our model with computer simulations is given in Sec. 6. The conclusions of this presentation and a critical discussion of the results are the subject of Sec. 7 .

\section{Microwave cavities and ballistic quantum dots}

Consider the system shown schematically in Fig. 1. It consists of a cavity connected to the external world by two waveguides, ideally of infinite length. We may think of performing a microwave experiment with such a device [5]. A wave is sent in from one of the waveguides, the result being a reflected wave in the same waveguide and a transmitted wave in the other. One given point inside the system receives the contribution from many multiply scattered waves -that have bounced from the inner surface of the cavity- giving rise to the "complex" interference pattern mentioned in the Introduction. An alternative interpretation of the same situation is that the outgoing wave has suffered the effect of many resonances that are present inside the cavity. In any case, the resulting wave function shows an appreciable sensitivity to changes in external parameters: for example, a change in the shape of the cavity may change the interference pattern drastically.

In experiments on electronic transport [6] performed with ballistic microstructures, or quantum dots, one finds that, for sufficiently low temperatures and for spatial dimensions on the order of $1 \mu \mathrm{m}$ or less, the phase coherence length $l_{\phi}$ exceeds the system dimensions, so that the phase of the single-electron wave function -in an independent-electron approximation- remains coherent across the system of interest. Under these conditions, these systems are called mesoscopic [7-9]. The elastic mean free path $l_{e l}$ also exceeds the system dimensions: impurity scattering can thus be neglected, so that only scattering from the boundaries of the

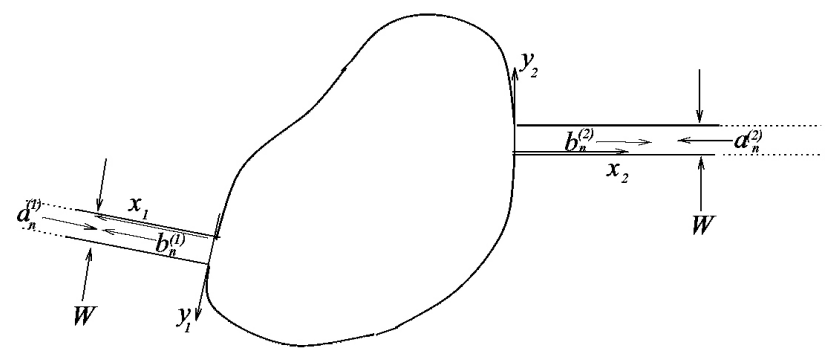

FIGURE 1 . The 2D cavity referred to in the text. The cavity is connected to the outside via two waveguides, each supporting $N$ running modes. The arrows inside the waveguides indicate incoming or outgoing waves. In waveguide $l=1,2$ the wave amplitudes are indicated as $a_{n}^{(l)}, b_{n}^{(l)}$, respectively, where $n=1, \cdots, N$. system is important. In these systems the dot acts as a resonant cavity and the leads as electron waveguides. Experimentally, an electric current is established through the leads that connect the cavity to the outside, the potential difference is measured and the conductance $G$ is then extracted. Assume that the microstructure is placed between two reservoirs (at different chemical potentials) shaped as expanding horns with negligible reflection back to the microstructure. In a picture that takes into account the electron-electron interaction in the random-phase approximation (RPA), the twoterminal conductance at zero temperature -understood as the ratio of the current to the potential difference between the two reservoirs- is then given by $[1,10]$

$$
\begin{aligned}
& G=2 \frac{e^{2}}{h} T \\
& T=t r\left(t t^{\dagger}\right),
\end{aligned}
$$

$t$ being the matrix of transmission amplitudes $t_{a b}$ associated with the resulting self-consistent single-electron problem at the Fermi energy $\epsilon_{F}$, with $a, b$ denoting final and initial channels. In this "scattering approach" to electronic transport, pioneered by Landauer [11], the problem is thus reduced to understanding the quantum-mechanical single-electron scattering by the cavity in question.

When the system is immersed in an external magnetic field $B$ and the latter is varied, or the Fermi energy $\epsilon_{F}$, or the shape of the cavity are varied, the relative phase of the various partial waves changes and so do the interference pattern and the conductance. This sensitivity of $G$ to small changes in parameters through quantum interference is called conductance fluctuations.

The experiments on quantum dots given in Ref. [6] report cavities in the shape of a stadium, for which the singleelectron classical dynamics would be chaotic, as well as experimental ensembles of shapes. Averages of the conductance, its fluctuations and its full distribution were obtained over such ensembles.

In what follows we shall phrase the problem in the language of electronic scattering; spin will be ignored, so that we shall deal with a scalar wave equation. However, our formalism is applicable to scattering of microwaves, or other classical waves, in situations where the scalar wave equation is a reasonable approximation.

\section{The scattering problem}

Very generally, a quantum-mechanical scattering problem is described by its scattering matrix, or $S$ matrix, whose rows and columns label the so called open channels.

In our application to mesoscopic physics we consider a system of noninteracting "spinless" electrons and study the scattering of an electron at the Fermi energy $\epsilon_{F}$ by a 2D microstructure, connected to the outside by two leads of width $W$ (see Fig. 1). 
The transverse modes, or channels, originate from the confinement of the electron in the transverse direction in the leads. If the incident Fermi momentum $k_{F}$ satisfies

$$
N<k_{F} W / \pi<N+1
$$

there are $N$ transmitting or running modes (open channels) in each of the two leads. The wavefunction in lead $l(l=1,2$ for the left and right leads, respectively) is written as the $N$ dimensional vector

$$
\Psi^{(l)}\left(x_{l}\right)=\left[\psi_{1}^{(l)}\left(x_{l}\right), \cdots, \psi_{N}^{(l)}\left(x_{l}\right)\right]^{T},
$$

the $m$-th component being a linear combination of unit-flux plane waves, i.e.

$$
\begin{aligned}
\psi_{m}^{(l)}\left(x_{l}\right) & =a_{m} \frac{\mathrm{e}^{-i k_{m} x_{l}}}{\left(h k_{m} / \mu\right)^{1 / 2}}+b_{m} \frac{\mathrm{e}^{i k_{m} x_{l}}}{\left(h k_{m} / \mu\right)^{1 / 2}}, \\
m & =1, \cdots, N
\end{aligned}
$$

In (4), $k_{m}$, the "longitudinal" momentum in channel $m$, is such that

$$
k_{m}^{2}+\left[\frac{m \pi}{W}\right]^{2}=k_{F}^{2}
$$

The $2 N$-dimensional $S$ matrix relates the incoming to the outgoing amplitudes as

$$
\left[\begin{array}{l}
b^{(1)} \\
b^{(2)}
\end{array}\right]=S\left[\begin{array}{l}
a^{(1)} \\
a^{(2)}
\end{array}\right],
$$

where $a^{(1)}, a^{(2)}, b^{(1)}, b^{(2)}$ are $N$-dimensional vectors. The matrix $S$ has the structure

$$
S=\left(\begin{array}{cc}
r & t^{\prime} \\
t & r^{\prime}
\end{array}\right)
$$

where $r, t$ are the $N \times N$ reflection and transmission matrices for particles from the left and $r^{\prime}, t^{\prime}$ for those from the right. This thus defines the transmission matrix $t$ used earlier in Eq. (1b).

The requirement of flux conservation (FC) implies unitarity of the $S$ matrix [1], i.e.

$$
S S^{\dagger}=I
$$

This is the only requirement in the absence of other symmetries. For a time-reversal-invariant (TRI) problem (as is the case in the absence of a magnetic field) and no spin, the $S$ matrix, besides being unitary, is symmetric [1]:

$$
S=S^{T} \text {. }
$$

\section{Doing statistics with the scattering matrix}

In numerical simulations of quantum scattering by $2 D$ cavities with a chaotic classical dynamics one finds that the $S$ matrix and hence the various transmission and reflection coefficients fluctuate considerably as a function of the incident energy $E$ (or incident momentum $k$ ), because of the resonances occurrying inside the cavity [1]. These resonances are moderately overlapping for just one open channel and become more overlapping as more channels open up. An example is illustrated in Fig. 2. One is led to a statistical analysis of the problem, just as was explained in the Introduction.

As $E$ changes, $S(E)$ wanders on the unitarity sphere, restricted by the condition of symmetry if TRI applies. The question we shall address is: what fraction of the time do we find $S$ inside the "volume element" $d \mu(S) ?(d \mu(S)$ is the invariant measure for $S$ matrices, to be described below.) We call $d P(S)$ this fraction and write it as

$$
d P(S)=p(S) d \mu(S)
$$

where $p(S)$ will be called the "probability density". Once we put the problem in this language we realize that we are dealing with a random $S$-matrix theory. In the next section we introduce a model to evaluate the probability density $p(S)$.

The concept of invariant measure $d \mu(S)$ for $S$ matrices mentioned above is an important one. By definition, the invariant measure for a given symmetry class does not change under an automorphism of that class of matrices unto itself $[13,14]$, i.e.

$$
\mathrm{d} \mu^{(\beta)}(S)=\mathrm{d} \mu^{(\beta)}\left(S^{\prime}\right)
$$

For unitary symmetric $S$ matrices we have

$$
S^{\prime}=U_{0} S U_{0}^{T}
$$

$U_{0}$ being an arbitrary, but fixed, unitary matrix: Eq. (12) represents an automorphism of the set of unitary symmetric

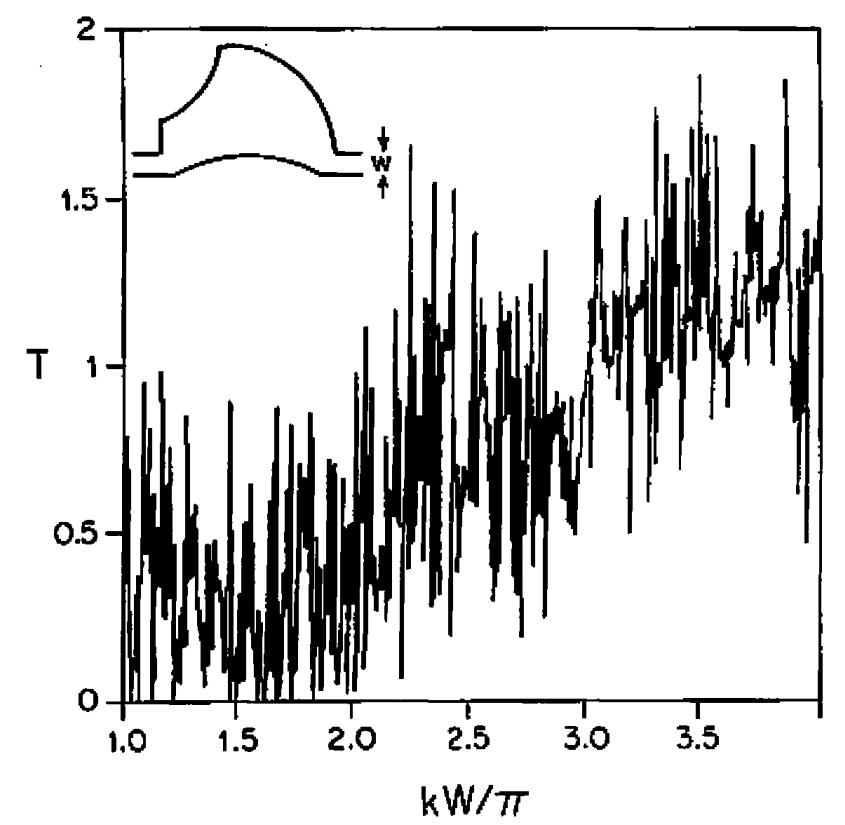

FIGURE 2. Total transmission coefficient of Eq. (1b) as a function of incident momentum, obtained by solving numerically the Schrödinger equation for the system indicated in the upper left corner (from Ref. 12). 
matrices unto itself. This is the orthogonal case, also known as the $\beta=1$ case, which we shall apply to our system when we ignore spin and in the presence of TRI. When TRI is broken, we deal with unitary, not necessarily symmetric, $S$ matrices. The automorphism is then

$$
S^{\prime}=U_{0} S V_{0},
$$

$U_{0}$ and $V_{0}$ being now arbitrary fixed unitary matrices. For this unitary, or $\beta=2$, class the resulting measure is the well known Haar's measure of the unitary group and its uniqueness is well known. Uniqueness for the $\beta=1$ class (and $\beta=4$, not discussed here) was shown in Ref. [13]. The invariant measure, Eq. (11), defines the so-called Circular (Orthogonal, Unitary) Ensemble (COE, $C U E$ ), for $\beta=1,2$ respectively.

\section{The maximum-entropy model}

Just as in the nuclear theory of the compound nucleus, we shall assume here too that in the scattering process by our cavities two distinct time scales occur [1,4,15-18]:

1. A prompt response arising from short paths. (In nuclear physics we would speak of direct processes.)

2. A delayed response arising from very long paths. (In nuclear physics we would speak of the equilibrated response, arising from the compound-nucleus formation.)

The prompt response is described mathematically in terms of $\bar{S}$, the average over an energy interval around a given energy $E$ of the actual scattering matrix $S(E)$ : these averaged, or optical, amplitudes vary much more slowly with energy than the original ones.

The statistical distribution of the scattering matrix $S(E)$ in our chaotic-scattering problem is constructed through a maximum-entropy "ansatz", assuming that it depends parametrically solely on the optical matrix $\bar{S}$, the rest of details being a mere "scaffolding".

As in the field of statistical mechanics, it is convenient to think of an ensemble of macroscopically identical cavities, described by an ensemble of $S$ matrices (see also Refs. 19 and 20). In statistical mechanics, time averages are very difficult to construct and hence are replaced by ensemble averages using the notion of ergodicity. Similarly, in the present context we idealize $S(E)$, for all real $E$, as a $s t a$ tionary random-matrix function of $E$ satisfying the condition of ergodicity [1,18], so that we may study energy averages in terms of ensemble averages. For instance, the optical matrix $\bar{S}$ will be calculated as an ensemble average, i.e., $\bar{S}=\langle S\rangle$.

We shall assume $E$ to be far from thresholds: locally, $S(E)$ is then a meromorphic function which is analytic in the upper half of the complex-energy plane and has resonance poles in the lower half plane.

From "analyticity-ergodicity" one can show the "reproducing” property: given an "analytic function" of $S$, i.e.,

$$
f(S)=\sum_{a_{1} b_{1} \cdots a_{p} b_{p}} c_{a_{1} b_{1} \cdots a_{p} b_{p}} S_{a_{1} b_{1}} \cdots S_{a_{p} b_{p}},
$$

we must have:

$$
\langle f(S)\rangle \equiv \int f(S) p_{\langle S\rangle}(S) d \mu(S)=f(\langle S\rangle) .
$$

This is the mathematical expression of the physical notion of analyticity-ergodicity. Thus $p_{\langle S\rangle}(S)$ must be a "reproducing kernel". Notice that only the optical matrix $\langle S\rangle$ enters the definition. The reproducing property (15) and reality of the distribution do not fix the ensemble uniquely. However, among the real reproducing ensembles, Poisson's kernel [14], i.e.,

$$
p_{\langle S\rangle}^{(\beta)}(S)=V_{\beta}^{-1} \frac{\left[\operatorname{det}\left(I-\langle S\rangle\left\langle S^{\dagger}\right\rangle\right)\right]^{(\beta n+2-\beta) / 2}}{\left|\operatorname{det}\left(I-S\left\langle S^{\dagger}\right\rangle\right)\right|^{\beta n+2-\beta}},
$$

is special, because its information entropy $[1,15]$

$$
\mathcal{S}\left[p_{\langle S\rangle}\right] \equiv-\int p_{\langle S\rangle}(S) \ln \left[p_{\langle S\rangle}(S)\right] d \mu(S)
$$

is greater than or equal to that of any other probability density satisfying the reproducibility requirement for the same optical $\langle S\rangle$. We could describe this result qualitatively saying that, for Poisson's kernel, $S$ is as random as possible, consistent with $\langle S\rangle$ and the reproducing property.

As for the information-theoretic content of Poisson's kernel, Eq. (16), we may say that Poisson's kernel describes a system with the following general properties:

i) unitarity of the $S$ matrix (flux conservation),

ii) analyticity of $S(E)$ implied by causality,

iii) presence or absence of time-reversal invariance (and spin-rotation symmetry when spin is taken into account) which determines the universality class: orthogonal $(\beta=1)$, unitary $(\beta=2)$ or symplectic $(\beta=4)$;

system-specific properties, parametrized by the ensemble average $\langle S\rangle$, which describes the presence of short-time processes. System-specific details other than the optical $\langle S\rangle$ are assumed to be irrelevant.

\section{Results of the information-theoretic model and comparison with numerical simulations}

A number of computer simulations have been performed, solving numerically the Schrödinger equation for $2 \mathrm{D}$ structures, in order to compare with our theoretical results. The optical $S$ was extracted from the numerical data and used as an input to Eq. (16), in order to find the distribution of $T$ from our model, which thus becomes a parameter-free prediction.

Some of the results are presented in Fig. 3 for a system with $N=1$ and embedded in a magnetic field, so that the relevant universality class is the unitary one $(\beta=2)$. The agreement between the numerical solutions of the Schrödinger equation and our maximum-entropy model is, generally 


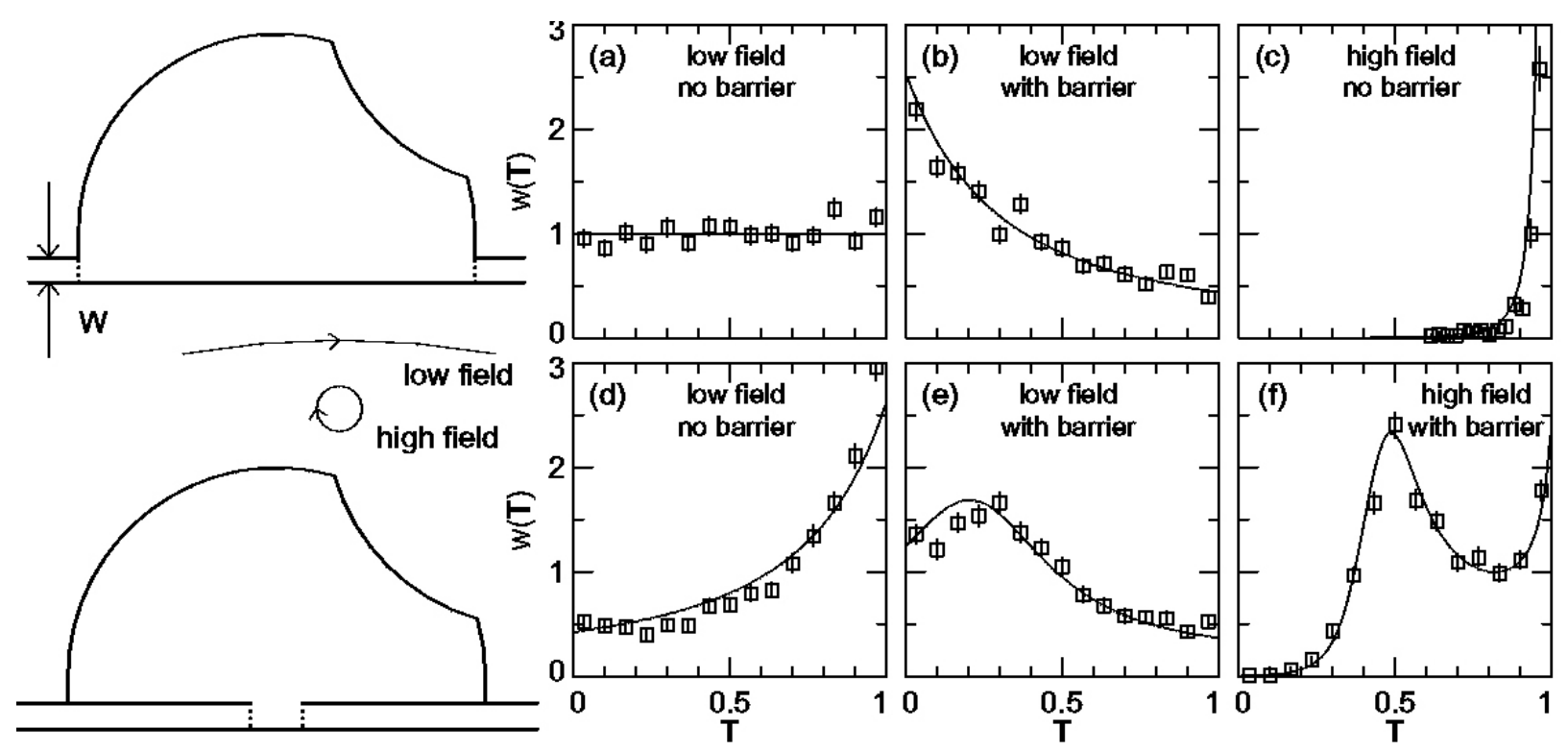

FIGURE 3. The probability density of the total transmission coefficient $T$ obtained integrating numerically the Schrödinger equation for the billiards shown on the left side of the figure for $N=1$. The results are indicated with squares, that include the statistical error bars. The curves are obtained from Poisson's kernel, Eq. (16), with $\langle S\rangle$ extracted from the numerical data. The agreement is very good. (From Ref. 16.)

speaking, found to be very good [16]. The magnetic field was increased as much as to produce a cyclotron radius smaller than a typical size of the cavity, and about twice the width of the leads, and the agreement was still found to be good.

In recent calculations [21] the effect of direct processes due to the presence of whispering gallery modes has been investigated. These modes were absent in the cavities illustrated in Fig. 3. Systems were studied for the orthogonal $(\beta=1)$ symmetry and with one and two channels $(N=1,2)$. The agreement between theory and numerical simulations is generally found to be good.

\section{Conclusions and Discussion}

In this contribution we reviewed a model that i) was originally developed in the realm of nuclear physics to describe statistical nuclear reactions when only direct processes and compound nucleus contributions are important, and ii) was then applied to the description of statistical scattering of oneparticle systems inside cavities whose classical dynamics is chaotic, and scattering of classical waves by cavities of a similar shape.

The above predictions for $w(T)$ in the presence of direct processes should be experimentally observable in microstructures where phase breaking is small enough, the sampling of the conductance distribution being performed by varying the energy or shape of the structure with an external gate voltage. They should also be observable in microwave scattering experiments.

We have reported on the comparison of the theoretical predictions with computer simulations. In both Refs. 16 and 21 the agreement was generally found to be good. In Ref. 21 we encountered cases in which it was not possible to find an energy interval $\Delta E$ in which approximate stationarity could be defined. (It is conceivable that this situation might be due to the presence of other time scales in the problem: this point remains to be investigated.) Yet, reducing $\Delta E$ literally to a point and collecting data over an ensemble constructed by changing the position of an obstacle inside the cavity, the agreement between theory and numerical experiment could be significantly improved, being excellent in several cases: in other words, Poisson's kernel seems to give a good description of the statistics of the data taken across the ensemble for a fixed energy. As a result, Poisson's kernel seems to be valid beyond the situation where it was originally derived, which required the properties of analyticity, stationarity and ergodicity, plus a maximum-entropy ansatz. It is as though the reproducing property of Eq. (15) were valid even in the absence of stationarity and ergodicity (analitycity is always there, of course). At the present moment we are unable to give an explanation of this fact. 
1. P.A. Mello and N. Kumar, Quantum Transport in Mesoscopic Systems, Complexity and Statistical Fluctuations (Oxford University Press, New York, 2004).

2. P. Sheng, Scattering and Localization of Classical Waves in Random Media, (World Scientific, Singapore, 1990).

3. T. Ericson and T. Mayer-Kuckuck Ann. Rev. Nucl. Sc. 16 (1966) 183.

4. H. Feshbach, C.E. Porter, and V.F. Weisskopf, Phys. Rev. 96 (1954) 448; H. Feshbach, Topics in the Theory of Nuclear Reactions, in Reaction Dynamics (Gordon and Breach, New York, 1973).

5. J. Stein, H.-J. Stöckmann, and U. Stoffregen, Phys. Rev. Lett. 75 (1995) 53 and references therein; A. Kudrolli, V. Kidambi, and S. Sridhar, Phys. Rev. Lett. 75 (1995) 822 and references therein; H. Alt et al., Phys. Rev. E 58 (1998) 1737 and references therein.

6. C. M. Marcus et al., Phys. Rev. Lett. 69 (1992) 506; Phys. Rev. B 48 (1993) 2460; A.M. Chang et al., Phys. Rev. Lett. 73 (1994) 2111; M.J. Berry et al., Phys. Rev. B 50 (1994) 17721; I.H. Chan et al., Phys. Rev. Lett. 74 (1995) 3876; M.W. Keller et al., Phys. Rev. B 53 (1996) R1693; Huibers et al., Phys. Rev. Lett. 81 (1998) 1917.

7. B.L. Altshuler, P.A. Lee, and R.A. Webb, eds., Mesoscopic Phenomena in Solids (North-Holland, Amsterdam, 1991).

8. C.W.J. Beenakker and H. van Houten, in Solid State Physics, edited by $\mathrm{H}$. Eherenreich and D. Turnbull, 44 (Academic, New York, 1991) 1

9. C.W.J. Beenakker, Rev. Mod. Phys. 69 (1997) 731.
10. M. Büttiker, IBM Jour. Res. Develop. 32 (1988) 317.

11. R. Landauer, Phil. Mag. 21 (1970) 863.

12. P.A. Mello and H.U. Baranger, Physica A 220 (1995) 15.

13. F.J. Dyson, Jour. Math. Phys. 3 (1962) 140.

14. L. K. Hua, Harmonic Analysis of Functions of Several Complex Variables in the Classical Domain, (Amer. Math. Soc., Providence RI, 1963).

15. P.A. Mello, P. Pereyra, and T.H. Seligman, Ann. Phys. (N.Y.) 161 (1985) 254; W.A. Friedman and P.A. Mello, Ann. Phys. (N.Y.) 161 (1985) 276.

16. H.U. Baranger and P. A. Mello, Europhys. Lett. 33 (1996) 465.

17. P.A. Mello and H.U. Baranger, Waves Random Media 9 (1999) 105.

18. P.A. Mello, Theory of Random Matrices: Spectral Statistics and Scattering Problems, in Mesoscopic Quantum Physics, E. Akkermans, G. Montambaux and J.-L. Pichard, eds. (Les Houches Summer School, Session LXI). See also references contained therein.

19. H.U. Baranger and P.A. Mello, Phys. Rev. Lett. 73 (1994) 142.

20. R.A. Jalabert, J.-L. Pichard, and C.W.J. Beenaker, Europhys. Lett. 27 (1994) 255; R.A. Jalabert and J.-L. Pichard, Journal de Physique I 5 (1995) 287; P. Brouwer and C.W.J. Beenaker, Phys. Rev. B 50 (1994) 11263; P. Brouwer, Phys. Rev. B 51 (1995) 16878.

21. E.N. Bulgakov, V.A. Gopar, P.A. Mello, and I. Rotter, Phys. Rev. B 73 (2006) 155302. 